\title{
Evolution of microdroplet morphology confined on asymmetric micropillar structures
}

\section{Binjian Ma ${ }^{1}$, Li Shan ${ }^{1}$, Baris Dogruoz ${ }^{2}$, Damena Agonafer ${ }^{1}$}

\author{
${ }^{1}$ Mechanical Engineering and Materials Science, Washington University in St. Louis, St. Louis, MO \\ 63130 \\ ${ }^{2}$ Cisco Systems Inc., 425 E Tasman Dr., San Jose, CA 95134
}

\section{Supporting Information}

\section{S.1 Fabrication process}

A fabrication flow chart for micropillar structures is shown in Figure S.1. A double-sided polished wafer wafer was first cleaned using RCA-1 silicon wafer cleaning method [1]. Plasma enhanced chemical vapor deposition (PECVD) was used to grow $1 \mu \mathrm{m} \mathrm{SiO} 2$ on both sides of the cleaned silicon wafer. Then photolithography and reactive-ion etching (RIE) was used to get a through-hole in the micropillar center. At last, the micropillar patterns were fabricated using photolithography and RIE.

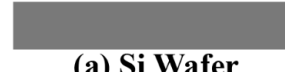

(a) Si Wafer
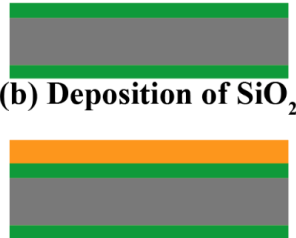

(c) Spin coating of photoresist

(d) Exposure and develop

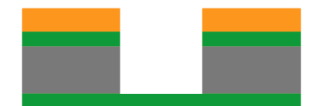

(e) RIE and DIRE of through holes

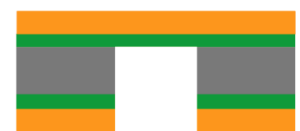

(f) Flip the sample and spin coating of photoresist

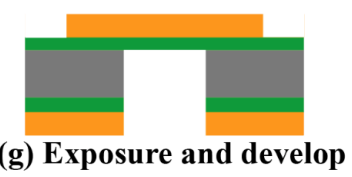

(h) RIE and DIRE of the pillars

(i) Remove all photoresit and RIE of $\mathrm{SiO}$

Figure S.1 Detailed microfabrication flow chart for micropillar structures 


\section{S.2 Experimental platform}

(a)

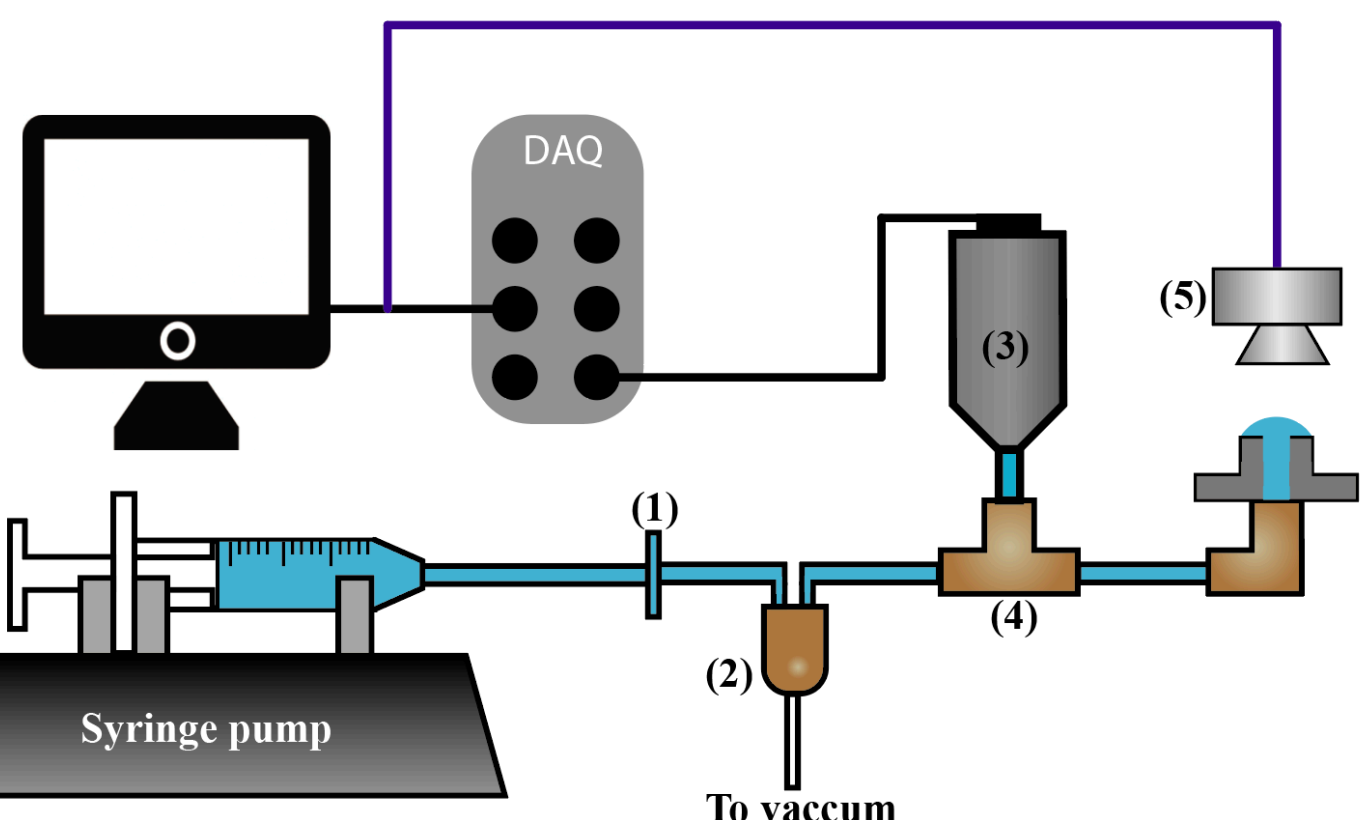

(b)

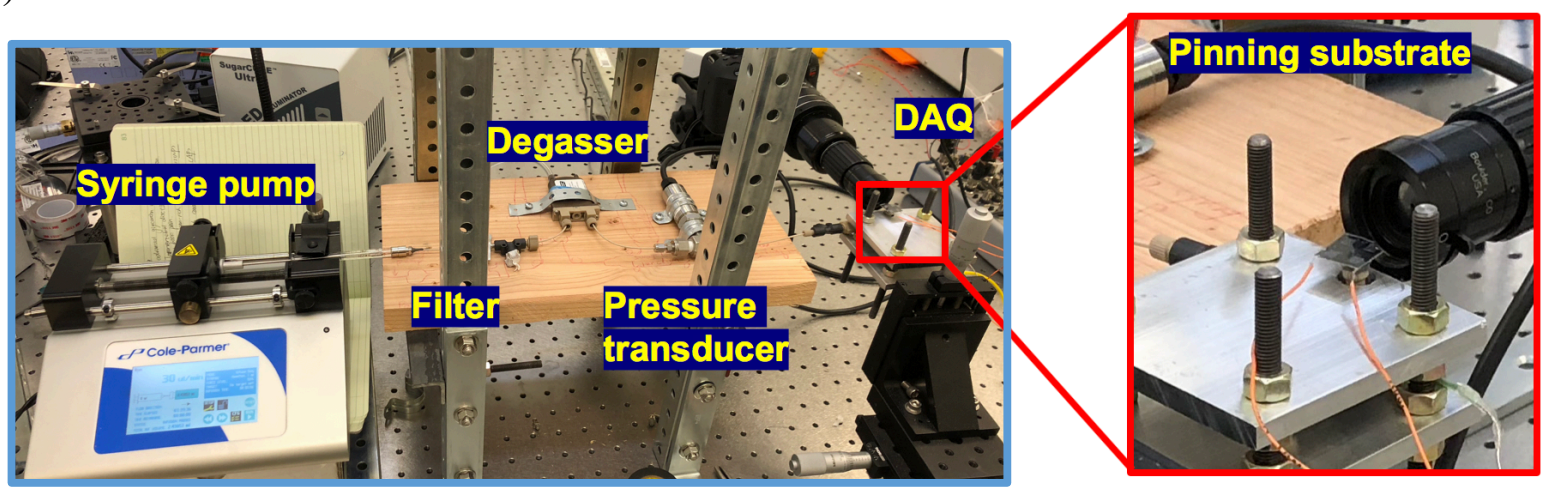

Figure S.2 (a) Schematic representation of the experimental setup. (1) particle filter, (2) minichamber degasser, (3) Pressure transducer, (4) Idex 3-way fitting, (5) camera. (b)Photograph of experimental platform setup for pinning experiments.

Figure S.2 illustrates is a photograph of our experimental platform for pinning experiments.

The experimental platform consists of three parts: a working liquid delivery system, a sample mounting platform, and a data acquisition system. Working liquid was delivered by a programmable syringe pump (Cole Parmer, syringe pump Touch Screen 100), a Hamilton 1 $\mathrm{mL}$ syringe, Idex tubing and fittings a 0.2 um particle filter (Idex, VHP-300) and a degasser (Idex, minichamber). Fabricated pillar samples were bonded to Idex nanoports using JB weld. Then pillar samples were fixed on a machined mount. The experimental data are collected 
using a DAQ (National Instrument, USB-6363). Microdroplets' images and videos are captured by a camera (Canon, Rebel Ti6) along with a microscopic lens.

When doing the experiments, the pumping rate for syringe pump was set and the working liquids will go through the particle filter, degasser and pressure transducer in serial, to remove the indissoluble particles, remove dissolved gases and measure the liquid pressure receptively. When a flat meniscus form at the micropillar tip, camera stated to record the videos for pinning behavior and the DAQ started to collect the pressure data.

\section{S.3 Characterization of the intrinsic contact angle}
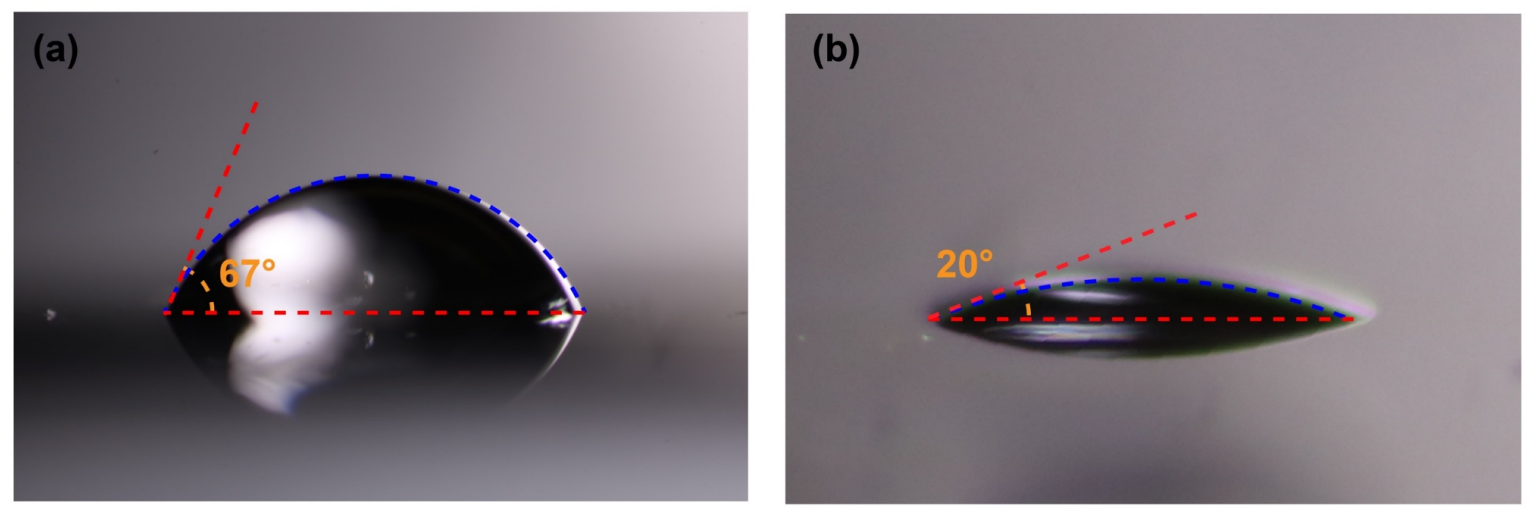

Figure S.3 (a) Contact angle of a water droplet resting on the silicon substrate; (b) Contact angle of an IPA solution $(70 \% \mathrm{w} / \mathrm{w})$ droplet resting on the silicon substrate.

The intrinsic contact angle of the three working fluids on silicon (100) wafer were measured using goniometer. Figure S.3 shows the images of a water droplet and an IPA solution (70\% $\mathrm{w} / \mathrm{w})$ droplet resting on the silicon substrate. The corresponding contact angles were measured to be $67^{\circ}$ and $20^{\circ}$, respectively. For Novec ${ }^{\mathrm{TM}} 7500$, the contact angle is not measurable since the liquid will spread spontaneously on the surface and form an extremely thin film. 


\section{S.4 Analysis of the geometric parameters of the droplet during the wicking, pivoting,}

\section{and free expansion stages}

(a)

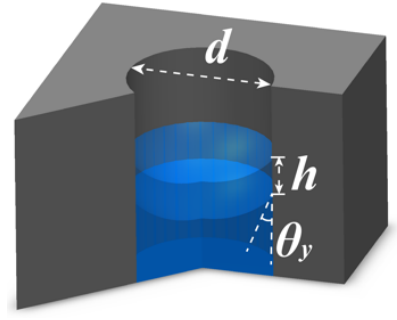

(b)

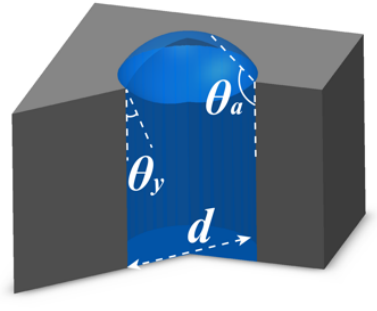

(c)

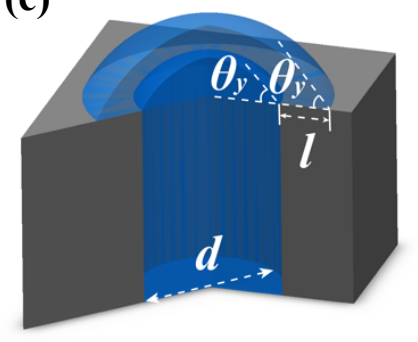

(d)

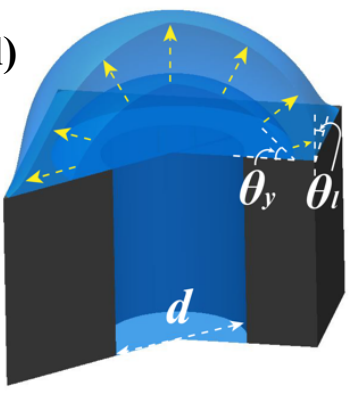

Figure S.4 Different stages of liquid advancing along the porous micropillar structure before bursting occurs: (a) the liquid wicks through the inner pore; (b) the meniscus pivots from a concave to a convex geometry; (c) the free expansion of the meniscus with a constant intrinsic contact angle on the top surface before the contact line advances to the outer edge of the micropillar; (d) the vertical growth of the droplet with its contact line partially pinned on the outer edge of the micropillar. Here, $d$ is the diameter of the pore at the center of the micropillar, $h$ is the increase in the height of the meniscus during the wicking stage, $l$ is the increase in the radius of the solid-liquid contact area during the free expansion stage, and $\theta_{l}$ is the local contact angle of the droplet during the pinning stage.

As described in the manuscript, the total free energy $E_{0}$ and pressure difference across the meniscus $\Delta p$ are calculated by

$$
\begin{aligned}
E_{0} & =\left(A_{s l}+A_{s a}\right) \gamma_{s a} \\
\Delta p=p_{\text {liquid }}-p_{\text {air }} & =\frac{d E_{t}}{d V_{l}}=\gamma_{l a}\left(\frac{d A_{l a}}{d V_{l}}-\cos \theta_{y} \frac{d A_{s l}}{d V_{l}}\right)
\end{aligned}
$$

1. Wicking through the inner pore (Figure S.4a): As liquid wicks from an initial height $z$ to

new height $z+h$, the changes in interfacial surface areas are given by:

$$
\begin{gathered}
\Delta A_{s l}=\pi d h \\
\Delta A_{l a}=0
\end{gathered}
$$

where $d$ is the diameter of the pore at the center of the micropillar and $h$ is the increase in the height of the meniscus. The change in liquid volume associated with increasing height $h$ is given by

Therefore,

$$
\Delta V_{\text {wicking }}=\frac{\pi}{4} d^{2} h
$$




$$
\begin{gathered}
\frac{\mathrm{d} A_{s l}}{\mathrm{~d} V_{l}}=\frac{\mathrm{d} A_{s l} / \mathrm{d} h}{\mathrm{~d} V_{l} / \mathrm{d} h}=\frac{\pi d}{\frac{\pi}{4} d^{2}}=\frac{4}{d} \\
\frac{\mathrm{d} A_{l a}}{\mathrm{~d} V_{l}}=0
\end{gathered}
$$

Substitute equations (3) - (4) into equation (1) and equations (6) - (7) into equation (2) gives

$$
\begin{aligned}
\Delta E_{\text {wicking }} & =-\pi d h \gamma_{l a} \cos \theta_{y} \\
\Delta p_{\text {wicking }} & =-\frac{4 \gamma_{l a} \cos \theta_{y}}{d}
\end{aligned}
$$

2. Pivoting from a concave to a convex meniscus (Figure S.4b): When the liquid meniscus forms a capped spherical curve with a contact angle of $\theta$ with the inner micropillar wall, the liquid-air interfacial area and the capped sphere volume are given by:

$$
\begin{gathered}
A_{l a}=\frac{\pi d^{2}}{2} \frac{1-\sin \theta}{\cos ^{2} \theta} \\
V_{c a p}=\frac{\pi d^{3}}{24}\left[\frac{(1-\sin \theta)^{2}(2+\sin \theta)}{\cos ^{3} \theta}\right]
\end{gathered}
$$

Therefore, the changes in interfacial areas when the contact angle increases from the intrinsic contact angle $\theta_{y}$ to an arbitrary apparent contact angle $\theta_{a}$ are given by:

$$
\begin{gathered}
\Delta A_{s l}=0 \\
\Delta A_{l a}=\frac{\pi d^{2}}{2}\left(\frac{1-\sin \theta_{a}}{\cos ^{2} \theta_{a}}-\frac{1-\sin \theta_{y}}{\cos ^{2} \theta_{y}}\right)
\end{gathered}
$$

From equations (16) and (17) we also have

$$
\begin{gathered}
\frac{\mathrm{d} A_{s l}}{\mathrm{~d} V_{l}}=0 \\
\frac{\mathrm{d} A_{l a}}{\mathrm{~d} V_{l}}=\frac{\mathrm{d} A_{l a} / \mathrm{d} \theta}{\mathrm{d} V_{l} / \mathrm{d} \theta}=-\frac{4 \cos \theta_{a}}{d}
\end{gathered}
$$

Substitute equations (18) - (19) into equation (6) and equations (20) - (21) into equation (8) gives

$$
\begin{gathered}
\Delta E_{\text {pivoting }}=\frac{\pi d^{2}}{2}\left(\frac{1-\sin \theta_{a}}{\cos ^{2} \theta_{a}}-\frac{1-\sin \theta_{y}}{\cos ^{2} \theta_{y}}\right) \gamma_{l a} \\
\Delta p_{\text {pivoting }}=-\frac{4 \gamma_{l a} \cos \theta_{a}}{d}
\end{gathered}
$$

3. Expanding freely on the top surface of micropillar before advancing to the outer edge

(Figure S.4c): When the apparent contact angle $\theta_{a}$ of the liquid meniscus with the top surface 
equals to the intrinsic contact angle, $\theta_{y}$, the meniscus will start to advance along the top pillar surface until it comes in contact with the outer edge. Assuming a capped spherical geometry is preserved, the changes in interfacial areas can be calculated as:

$$
\begin{gathered}
\Delta A_{s l}=\pi\left(l^{2}+d l\right) \\
A_{l a}=\frac{\pi}{2} \frac{(d+2 l)^{2}\left(1-\cos \theta_{y}\right)}{\sin ^{2} \theta_{y}} \\
\Delta A_{l a}=2 \pi\left(l^{2}+d l\right) \frac{1-\cos \theta_{y}}{\sin ^{2} \theta_{y}}
\end{gathered}
$$

where $l$ is the increase in the radius of the solid-liquid contact area as shown in Figure 2. The change in liquid volume can be calculated as:

$$
V_{\text {cap }}=\frac{\pi\left(1-\cos \theta_{y}\right)^{2}\left(2+\cos \theta_{y}\right)}{24 \sin ^{3} \theta_{y}}(d+2 l)^{3}
$$

From equations (24), (26) and (27) we have

$$
\begin{aligned}
\frac{\mathrm{d} A_{s l}}{\mathrm{~d} V_{l}} & =\frac{\mathrm{d} A_{s l} / \mathrm{d} l}{\mathrm{~d} V_{l} / \mathrm{d} l}=\frac{4 \sin ^{3} \theta_{y}}{\left(1-\cos \theta_{y}\right)^{2}\left(2+\cos \theta_{y}\right)(d+2 l)} \\
\frac{\mathrm{d} A_{l a}}{\mathrm{~d} V_{l}} & =\frac{\mathrm{d} A_{l a} / \mathrm{d} l}{\mathrm{~d} V_{l} / \mathrm{d} l}=\frac{8 \sin \theta_{y}}{\left(1-\cos \theta_{y}\right)\left(2+\cos \theta_{y}\right)(d+2 l)}
\end{aligned}
$$

Substitute equations (24) - (26) into equation (6) and equations (28) - (29) into equation (8) gives

$$
\begin{gathered}
\Delta E_{\text {expanding }}=\pi\left(l^{2}+d l\right)\left(\frac{2-2 \cos \theta_{y}}{\sin ^{2} \theta_{y}}-\cos \theta_{y}\right) \gamma_{l a} \\
\Delta p_{\text {expanding }}=\frac{4 \sin \theta_{y}}{d+2 l}
\end{gathered}
$$




\section{S.5 Modeling of equilibrium droplet geometry using Surface Evolver}

\begin{tabular}{l|l|l|l}
\hline $\begin{array}{c}\text { Droplet } \\
\text { volume (nL) }\end{array}$ & 3D-view & $\begin{array}{c}\text { 2D view } \\
\text { (normal to the side) }\end{array}$ & $\begin{array}{c}\text { 2d view } \\
\text { (normal to the diagonal) }\end{array}$ \\
\hline 4.25 & & & \\
\hline 106.31 & & & \\
\hline 637.85 & & & \\
\hline
\end{tabular}

Figure S.5 Profiles of droplets confined on triangular micropillar with different volumes taken from three different angles. The area of the top surface of the triangular micropillar is set as 1 .

An example of the morphologies of a series of equilibrated water droplets confined on a triangular micropillar structure with increasing droplet volume is given in Figure S.5. The intrinsic contact angle was set as $67^{\circ}$ and the side length of the triangular micropillar is fixed at $421 \mu \mathrm{m}$. As shown in the figure, as the contact line of the droplet reaches the outer edge of the triangular substrate, the droplet first starts to evolve into an irregular shape possessing both the feature of a capped sphere and a pyramid. In particular, the meniscus exhibits a sharper curve along the directions pointing from the droplet top towards the corner of the triangular substrate. Such features resemble the sharp interface between two adjacent faces in a pyramid geometry. The $2 \mathrm{D}$ view of the asymmetric droplet also reveals that the apparent local contact angle is much larger at the center of the sides compared to that at the corner of the triangular substrate. This suggests that the spilling behavior will eventually initiate from the center of the 
sides. As the volume of the droplet becomes larger, it can also be observed from the droplet profiles that the mean radius of curvature of the droplet decreases first and increases later until spilling occurs. The minimum radius of the curvature is obtained when the droplet profile becomes similar to a hemisphere. 
S.6 Impact of local geometry (corner fillet) on the wetting and pinning characteristics

\begin{tabular}{|c|c|c|c|c|c|}
\hline & $\mathrm{V}=0.01 \mathrm{~mm}^{3}$ & $\mathrm{~V}=0.02 \mathrm{~mm}^{3}$ & $\mathrm{~V}=0.03 \mathrm{~mm}^{3}$ & $\mathrm{~V}=0.04 \mathrm{~mm}^{3}$ & $\mathrm{~V}=0.05 \mathrm{~mm}^{3}$ \\
\hline $\begin{array}{c}\text { Triangular } \\
\text { micropillar } \\
\text { with sharp } \\
\text { corner }\end{array}$ & $148 \mathrm{~mm}^{2}$ & $\begin{array}{l}9 \mathrm{~mm}^{2} \\
0 \mathrm{~mm}^{2}\end{array}$ & $\begin{array}{l}392 \mathrm{~mm}^{2} \\
151 \mathrm{~mm}^{2}\end{array}$ & $\begin{array}{l}A_{l v}=0.480 \mathrm{~mm}^{2} \\
A_{s l}=0.151 \mathrm{~mm}^{2}\end{array}$ & $\begin{array}{l}A_{t v}=0.564 \mathrm{~mm}^{2} \\
A_{s l}=0.151 \mathrm{~mm}^{2}\end{array}$ \\
\hline $\begin{array}{c}\text { Triangular } \\
\text { micropillar } \\
\text { with rounded } \\
\text { corner }\end{array}$ & $\begin{array}{l}A_{l v}=0.204 \mathrm{~mm}^{2} \\
A_{s l}=0.148 \mathrm{~mm}^{2}\end{array}$ & $\begin{array}{l}A_{l v}=0.297 \mathrm{~mm}^{2} \\
A_{s l}=0.148 \mathrm{~mm}^{2}\end{array}$ & $\begin{array}{l}A_{l v}=0.390 \mathrm{~mm}^{2} \\
A_{s l}=0.148 \mathrm{~mm}^{2}\end{array}$ & $\begin{array}{l}A_{l v}=0.478 \mathrm{~mm}^{2} \\
A_{s l}=0.148 \mathrm{~mm}^{2}\end{array}$ & $\begin{array}{l}A_{l v}=0.561 \mathrm{~mm}^{2} \\
A_{s l}=0.148 \mathrm{~mm}^{2}\end{array}$ \\
\hline $\begin{array}{l}\text { Percentage } \\
\text { difference in } A_{t}\end{array}$ & $0.29 \%$ & $0.64 \%$ & $0.56 \%$ & $0.50 \%$ & $0.39 \%$ \\
\hline $\begin{array}{l}\text { Percentage } \\
\text { difference in } A_{s}\end{array}$ & $0.47 \%$ & $1.47 \%$ & $1.79 \%$ & $1.99 \%$ & $2.12 \%$ \\
\hline
\end{tabular}

Figure S.6 Profiles of droplets with different volumes confined on triangular micropillars with sharp and rounded corner features. The radius of the rounded corner is $50 \mu \mathrm{m}$. The results show that the inclusion of rounded corner does not have a significant effect on the droplet shape as well as the liquid-vapor and solid-liquid interfacial area.

To investigate if the introduction of corner fillet to the micropillar structure will affect the wetting and pinning characteristics of the droplet, we used Surface Evolver to analyze the equilibrium droplet profiles with a volume of $0.01,0.02,0.03,0.04$, and $0.05 \mathrm{~mm}^{3}$ on a triangular micropillar structure with and without the rounded corner feature, as shown in Figure S.6. The dimension of the triangular micropillar used in Surface Evolver matches exactly with the length scales shown in Figure 1 in the main manuscript. Based on the equilibrium droplet profiles, we calculated and compared the liquid-vapor and solid-liquid interfacial area of the droplets at five different volumes on the two different triangular micropillar structures. The results show that the maximum difference in the interfacial area for the droplet at the same volume is less than $3 \%$. Therefore, the local geometry (i.e., the rounded corner feature) does not have a significant impact on the wetting and pinning characteristic. 


\section{S.7 Top view snapshots of microdroplet expanding on a square micropillar structure}
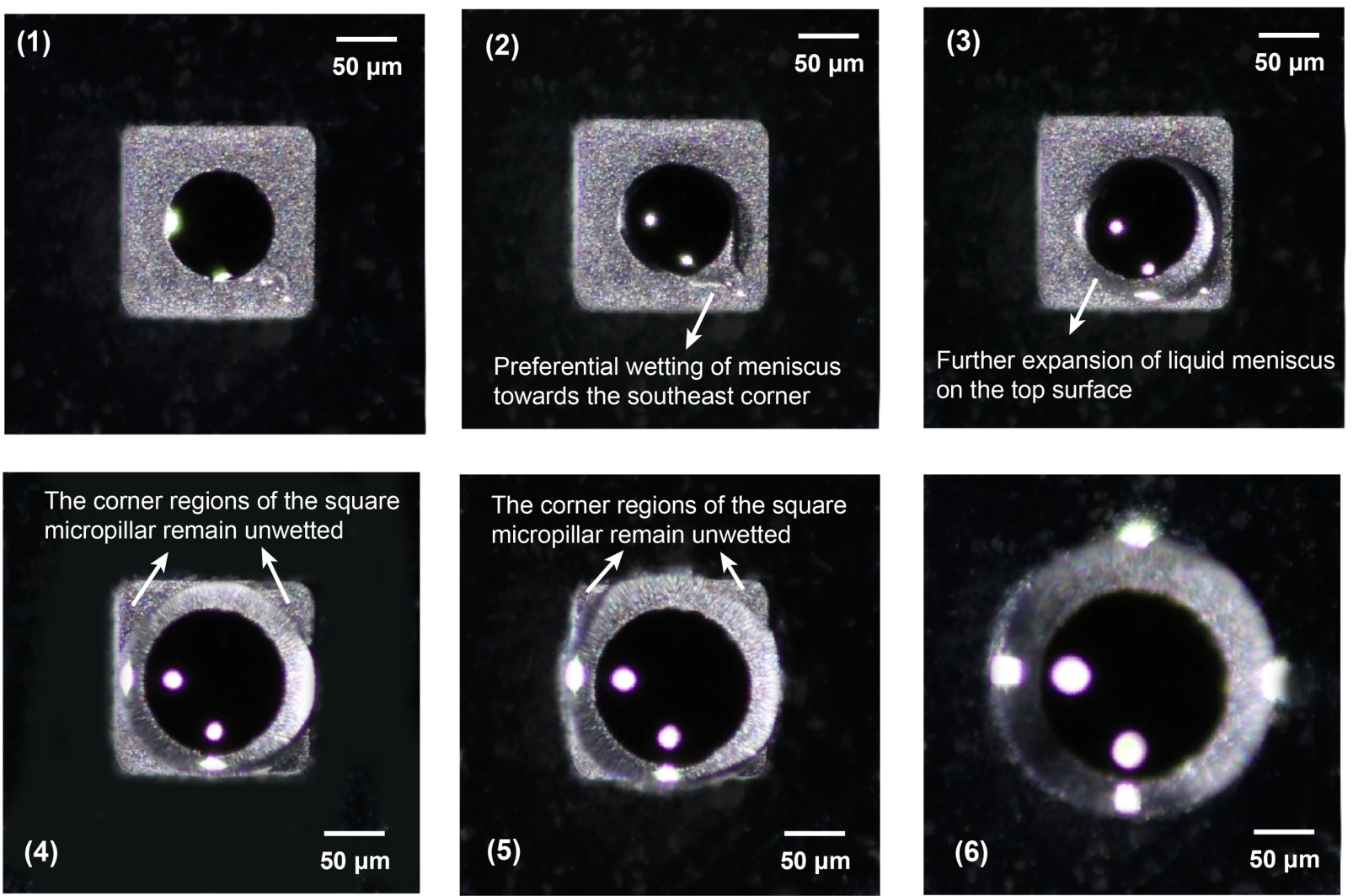

Figure S.7 Top view snapshots of a DI water droplet growing on a square micropillar at different stages: (1) the meniscus just emerges from the central pore; (2) the meniscus starts to spread from the central pore to the surrounding area on the top surface; (3) further free expansion of the meniscus on the top surface; (4) the contact line comes in contact with the outer edge of the micropillar; (5) the contact line is partially pinned along the outer edge and partially expanding towards the corner region of the micropillar; (6) the entire top surface of the micropillar is blocked from the top view by the droplet. During the expansion of the meniscus, the area near the center of the side edge gets covered by the droplet much faster than the area near the corner regions of the micropillar. Before the entire top surface is blocked by the droplet, a significant portion of the top surface near the corner regions still remains unwetted. (Note: the enlargement of the central pore from snapshot 1 to 4 is induced by the refraction of light in the liquid).

Figure S.7 shows the top view snapshots of the droplet taken during the expansion of the droplet on a square micropillar. After the liquid emerges on the top surface, the droplet meniscus initially finds its preferential wetting direction towards which the contact line spreads, as shown in Figure S.7(2). This preferential spreading is attributed to a non-homogeneous roughness on the top surface of the micropillar, which presents locally different energy barriers to the spreading of the contact line [2-7]. 


\section{S.8 AFM image of the square micropillar structure taken at different locations}

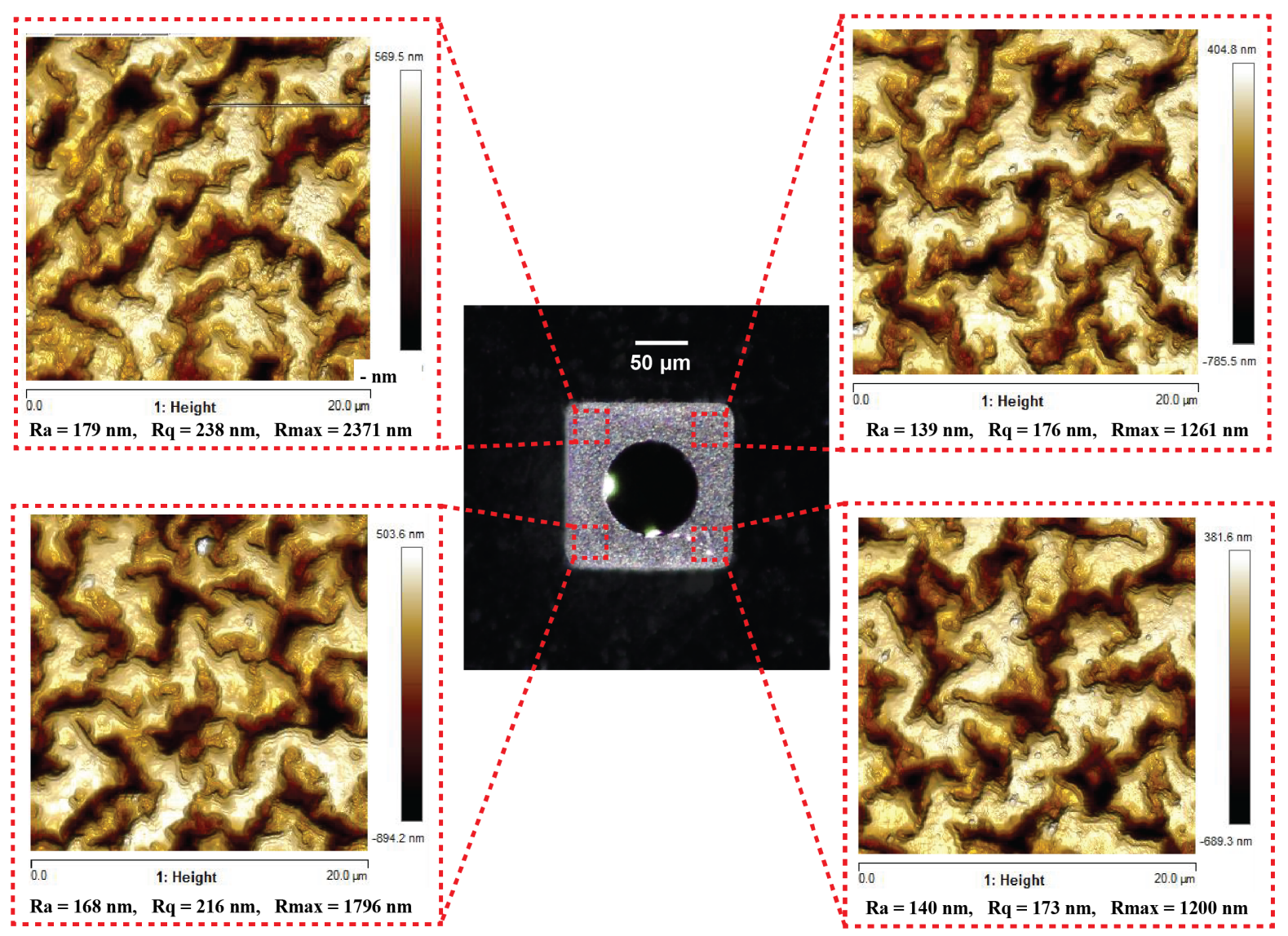

Figure S.8 AFM images taken at four different locations on the square micropillar. The largest and smallest roughness are found on the top left and bottom right corners of the micropillar, respectively.

Figure S.8 shows the AFM images of the square micropillar taken at four different locations near the corners. It is evident to notice that the surface roughness at the top left corner is significantly greater than that at the bottom right corner of the square micropillar sample. In particular, the maximum roughness depth at the top left corner is almost two times of that at the bottom right corner. At the microscale, the non-uniform surface topology creates geometric edge features which give rise to varied local pinning behavior and contact line hysteresis. Such principle has also been applied to design of superoleophobic surfaces with microstructure patterns (e.g., micropillars and micromeshes) [8-11]. Afterwards, further increase in the droplet volume causes the contact line to expand in other directions and eventually cover the majority of the top surface (shown in Figure S.7(4)). However, the contact line ceases to wet the corner 
region of the square micropillar as the droplet grows in volume (shown in Figure S.7(5)). In other words, the droplet is now expanding with its contact line partially pinned on the outer edge and partially remaining free on the top surface. Therefore, with a further increase in the droplet volume, the local apparent contact angle, $\theta_{a}$, increases sharply near the center of the outer edge, where the contact line is pinned, but remains relatively unchanged near the corner regions on the top surface, where the contact line can still move freely. Such behavior results in an asymmetric droplet profile which exhibits a larger curvature than a capped spherical droplet with the same contact perimeter and volume. 


\section{S.9 Top view snapshots of microdroplet expanding on a triangular micropillar structure}
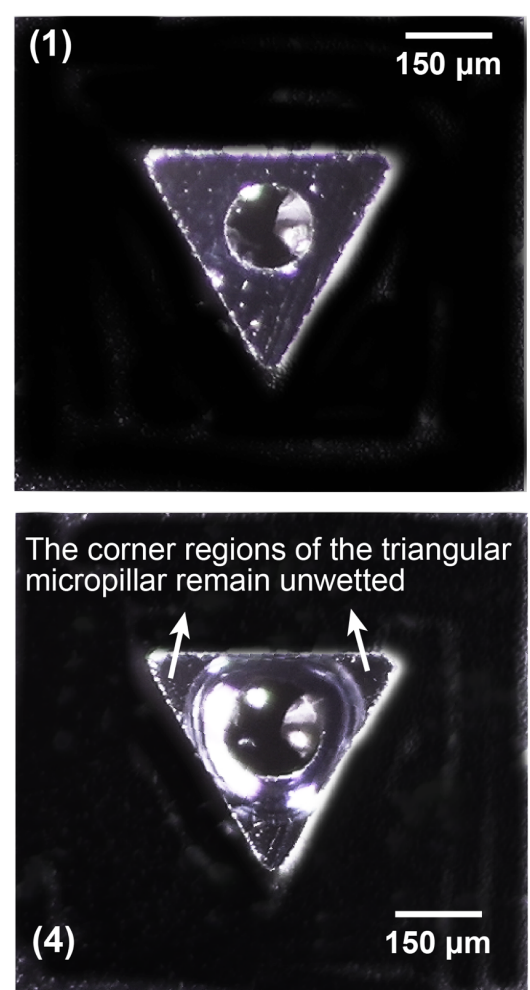
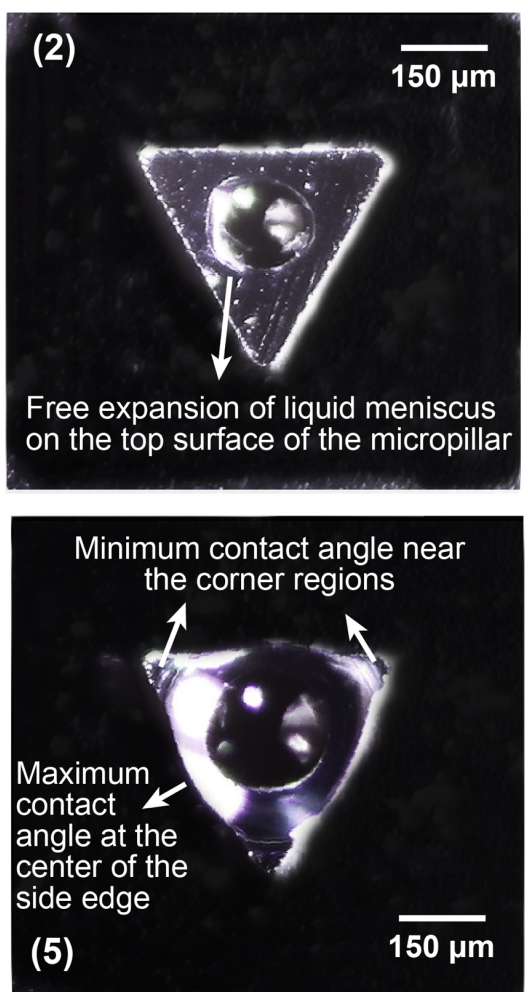
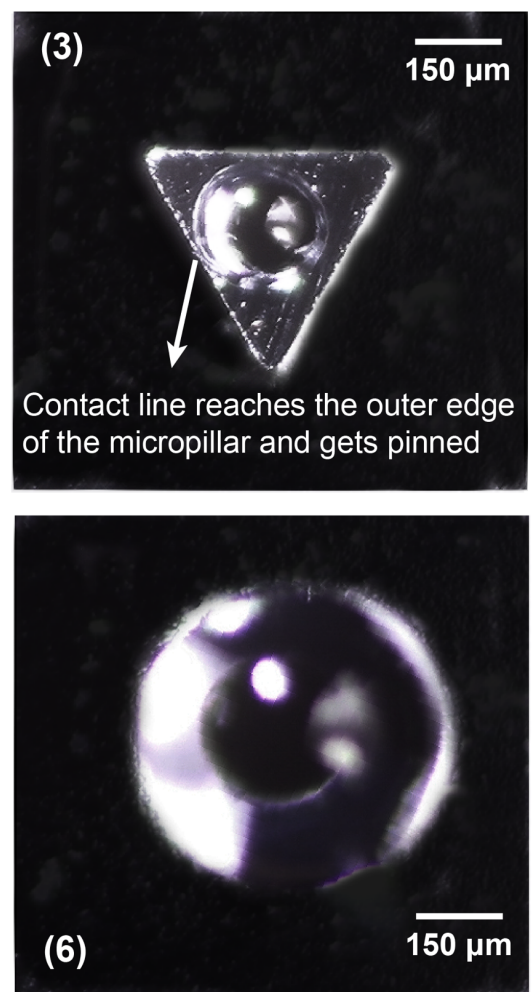

Figure S.9 Top view snapshots of a DI water droplet growing on a triangular micropillar at different stages: (1) the meniscus emerges from the central pore of the micropillar; (2) the meniscus starts to spread from the central pore to the surrounding area on the top surface of the micropillar; (3) the contact line of the droplet comes in contact with the outer edge of the micropillar; (4) the contact line is partially pinned along the outer edge and partially expands towards the corner region of the micropillar; (5) the continuous expansion of the meniscus with a greater fraction of the top surface being wetted by the droplet; (6) the entire top surface of the micropillar is blocked from the top view by the droplet. The behavior of the meniscus expanding on the triangular micropillar is very similar to that on a square micropillar structure. Specifically, the area near the center of the side edge gets wetted by the droplet much faster than the area near the corner regions of the micropillar. In addition, a significant portion of area near the corner regions of the micropillar remains unwetted before the entire top surface is blocked by the droplet.

Top view snapshots of a droplet advancing on a triangular micropillar are shown in Figure S.9.

Similar to the features shown in Figure S.7, the droplet meniscus first expands freely on the top surface with a non-isotropic spreading rate, due to the non-homogeneous roughness as previously discussed. As soon as the contact line comes in contact with the outer edge of the micropillar structure, further advancement beyond the micropillar is prohibited by the sharp edge feature, and therefore the meniscus starts to expand towards the corner regions of the 
micropillar structure. During this process, the shape of the droplet meniscus gradually transforms from a capped sphere to an irregular geometry. In particular, the local contact angle, $\theta_{y}$, starts to increase at different rates at different locations along the contact line. Specifically, near the center of the side edge, the advancement of the contact line is stopped by the sharp edge feature, which results in liquid accumulation in the vertical direction. Therefore, the largest contact angle is observed near the center of the micropillar side edge. On the contrary, the spreading of the liquid meniscus is unbounded near the corner region, which results in a flat meniscus profile and a smaller local contact angle at these locations. This feature is represented in Figure S.9(5). Comparing the droplet profile in Figure S.9 with that in Figure S.7, it is observed that the droplet growing on the triangular micropillar exhibits a greater level of asymmetry than that growing on the square micropillar. Therefore, the curvature of the meniscus is greater for the droplet growing on the triangular micropillar, resulting in a smaller critical volume compared to droplets pinned on circular and square micropillars. 
S.10 Experimental pressure traces of water droplets expanding on the circular, square, and triangular micropillar

(a)

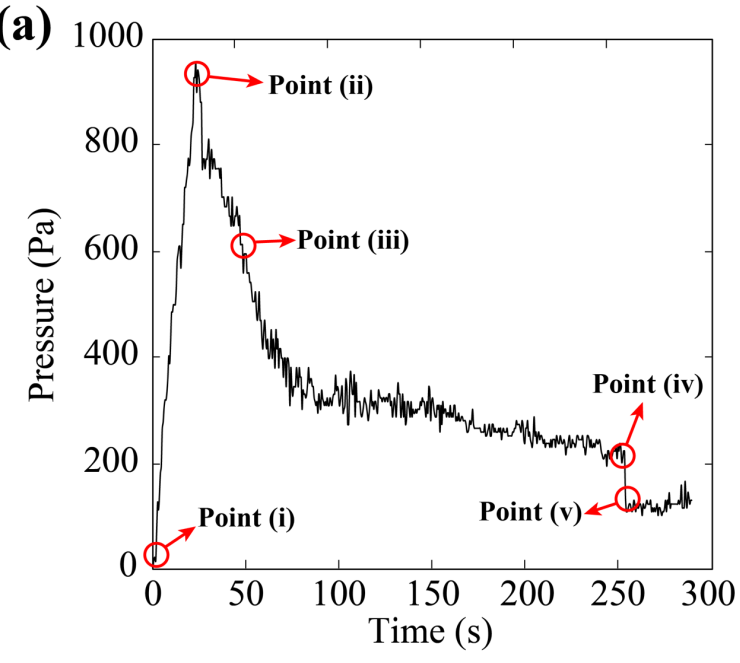

(b)

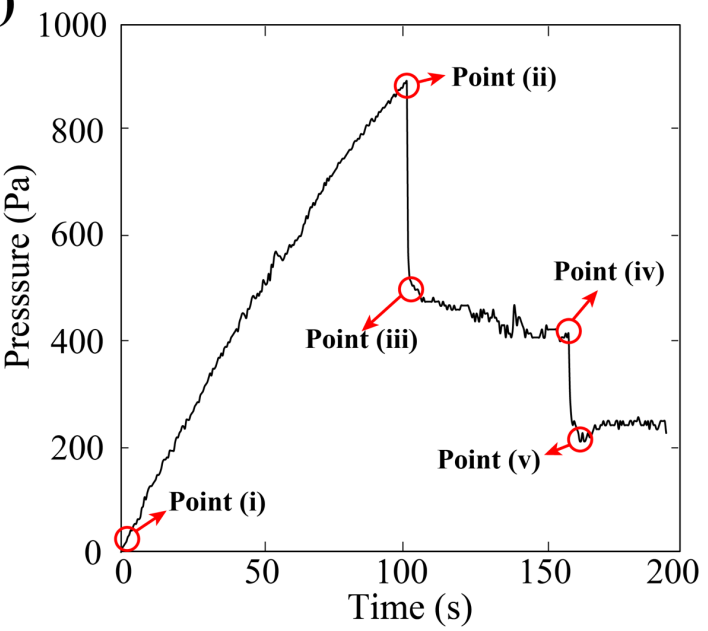

(c)

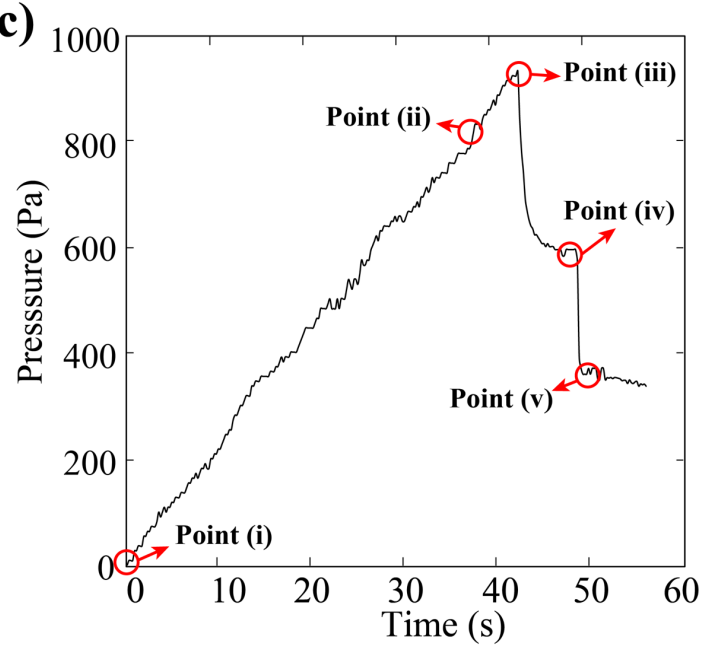

Figure S.10 Pressure curve during the growth of the DI water droplet on the (a) circular, (b) square, and (c) triangular micropillar. Points (i) to (v) corresponds to the moments when the snapshots in Figure 4 in the main manuscript were taken. A global maximum pressure is observed at point (ii) for the circular and square micropillar and at point (iii) for the triangular micropillar. Two drastic pressure drops are observed during the entire droplet expansion process. The first pressure drop is caused by the expansion of the droplet beyond a hemisphere. The second pressure droplet corresponds to spilling of the droplet over the outer edge of the micropillar. 


\section{Reference:}

[1] W. Kern, "Cleaning solution based on hydrogen peroxide for use in silicon semiconductor technology," RCA review, vol. 31, pp. 187-205, 1970.

[2] C. Dorrer and J. Rühe, "Contact line shape on ultrahydrophobic post surfaces," Langmuir, vol. 23, no. 6, pp. 3179-3183, 2007.

[3] Y. V. Kalinin, V. Berejnov, and R. E. Thorne, "Contact line pinning by microfabricated patterns: effects of microscale topography," Langmuir, vol. 25, no. 9, pp. 5391-5397, 2009.

[4] A. T. Paxson and K. K. Varanasi, "Self-similarity of contact line depinning from textured surfaces," Nature communications, vol. 4, p. 1492, 2013.

[5] L. Hu, M. Wu, W. Chen, H. Xie, and X. Fu, "Discontinuous pinning effect by a hole row to the gas-liquid interface in a parallel gap," Experimental Thermal and Fluid Science, vol. 87, pp. 50-59, 2017.

[6] P. S. Forsberg, C. Priest, M. Brinkmann, R. Sedev, and J. Ralston, "Contact line pinning on microstructured surfaces for liquids in the Wenzel state," Langmuir, vol. 26, no. 2, pp. 860-865, 2009.

[7] R. Seemann, M. Brinkmann, E. J. Kramer, F. F. Lange, and R. Lipowsky, "Wetting morphologies at microstructured surfaces," Proceedings of the National Academy of Sciences of the United States of America, vol. 102, no. 6, pp. 1848-1852, 2005.

[8] K. Kurogi, H. Yan, and K. Tsujii, "Importance of pinning effect of wetting in super water-repellent surfaces," Colloids and Surfaces A: Physicochemical and Engineering Aspects, vol. 317, no. 1-3, pp. 592-597, 2008.

[9] J. Berthier et al., "On the pinning of interfaces on micropillar edges," Journal of colloid and interface science, vol. 338, no. 1, pp. 296-303, 2009.

[10] J. Yeo, M. J. Choi, and D. S. Kim, "Robust hydrophobic surfaces with various micropillar arrays," Journal of Micromechanics and Microengineering, vol. 20, no. 2, p. 025028, 2010.

[11] R. Hensel et al., "Wetting resistance at its topographical limit: the benefit of mushroom and serif T structures," Langmuir, vol. 29, no. 4, pp. 1100-1112, 2013. 